\title{
Efecto del microhábitat sobre la abundancia y riqueza específica de los macroinvertebrados bentónicos en dos ríos tropicales de montaña, Guatemala
}

\author{
Effect of microhabitat on the abundance and specific richness of benthic \\ macroinvertebrates in two tropical mountain streams, Guatemala \\ Gabriela Dávila-Recinos ${ }^{1 *}$, José R. Ortíz-Aldana ${ }^{1}$, Fátima Reyes-Morales² \\ ${ }^{1}$ Instituto de Investigaciones Hidrobiológicas, Centro de Estudios del Mar y Acuicultura (Cema), \\ Universidad de San Carlos de Guatemala (Usac). \\ ${ }^{2}$ Departamento de Investigación y Calidad Ambiental (Dica), Autoridad para el manejo sustentable \\ de la cuenca del lago de Atitlán y su entorno (Amsclae). \\ *Autor al que se dirige la correspondencia: gabydavila_91@hotmail.com
}

Recibido: 11 de septiembre 2018 / Revisión: 25 de febrero 2019 / Aceptado: 25 de abril 2019

\section{Resumen}

os macroinvertebrados bentónicos cumplen funciones ecológicas importantes en los sistemas lóticos, como
el traspaso de energía a través de las redes tróficas, la descomposición de la materia orgánica, el reciclaje de
nutrientes y la mezcla de sedimentos. Uno de los principales factores que definen la composición del ensamble
de macroinvertebrados es la disponibilidad de microhábitats. Debido a ello, se evaluó el efecto de ocho microhá-
bitats (hojarasca, grava, piedras, roca, musgo, vegetación acuática, pozas y cataratas), así como de la velocidad y
profundidad, sobre la abundancia y riqueza específica de los macroinvertebrados en los ríos La Labor y Tzununá
en la cuenca del lago Atitlán en el altiplano de Guatemala. Se colectaron 19,529 organismos correspondientes a 91
taxa, 49 familias y 65 géneros. El microhábitat que presentó mayor abundancia fue hojarasca (7,723 organismos)
y pozas presentó la menor ( 652 organismos). En cuanto a riqueza, piedras presentó la mayor riqueza (41 taxa) y
musgo tuvo la menor ( 28 taxa). Se evidenció que el microhábitat tuvo un efecto significativo sobre la abundancia
y la riqueza ( $p<.05)$, ésta última también se vio influenciada por la profundidad ( $p=.030$ ). Se concluyó que el
microhábitat es un factor importante, para determinar la composición de la comunidad de macroinvertebrados; ya
que la presencia y dominancia de elementos de heterogeneidad en los ríos, contribuye a incrementar la diversidad
del hábitat físico, así como las opciones de alimento y refugio, promoviendo la abundancia y riqueza de macroin-
vertebrados en los sistemas lóticos.

Palabras claves: Macroinvertebrados Hojarasca, Piedras, Heterogeneidad

\section{Abstract}

$\mathrm{B}$ enthic macroinvertebrates serve important ecological functions in lotic systems, such as the transfer of energy through trophic networks, decomposition of organic matter, nutrient recycling and sediment mixing. One of the main factors that define the composition of the macroinvertebrate assemblage is the availability of microhabitats. The survey evaluated the effect of eight microhabitats (leaf litter, gravel, stones or pebbles, rock, moss, aquatic vegetation, pools and waterfalls), as well as speed and depth, on the abundance and specific richness due to rarefaction of the macroinvertebrates in La Labor and Tzununá rivers, which are part of the Lake Atitlán basin in the Guatemalan highlands. In this area 19,529 organisms corresponding to 91 taxa, 49 families and 65 genera were collected. The highest abundance was in leaf litter microhabitat (7,723 organisms), while stones showed the highest richness (41 taxa). Pools had the lowest abundance (652 organisms) and moss had the lowest richness (28 taxa). The microhabitat has a significant effect on abundance and richness $(p<.05)$, also depth has a significant effect on richness $(p=.030)$. The microhabitat is an important factor, to determine the composition of the macroinvertebrate community, because the presence and dominance of elements of heterogeneity in the rivers contributes to increase the diversity of the physical habitat, which provides refuge and food that satisfy the requirements of organisms promoting the abundance and richness of macroinvertebrates in lotic systems. 


\section{Introducción}

Los macroinvertebrados bentónicos son organismos cuya vida, o parte de ella se desarrolla en el agua, estos incluyen principalmente insectos (tricópteros, efímeras, moscas, escarabajos, chinches de agua, libélulas, entre otros), anélidos (lombrices de agua y sanguijuelas), moluscos y crustáceos. Estos son importantes en la ecología fluvial debido a su respuesta a perturbaciones en el ecosistema (Alonso \& Camargo, 2005), también forman parte de las redes tróficas, descomposición de materia orgánica, reciclaje de nutrientes y mezcla de sedimentos (Covich, Palmer, \& Crowl, 1999). Dentro de los ríos se encuentran sustratos, los cuales proveen soporte, permiten el desplazamiento, son lugar de interacción con otros organismos y reproducción de adultos, entre otros (Jiménez-Sandoval, 2015).

Uno de los factores que define la estructura del ensamble de macroinvertebrados bentónicos en ríos neotropicales es el medio físico, el cual se compone del tipo de hábitat que a su vez determina la calidad ecológica de los ecosistemas. Se considera que los sitios de mayor elevación y zonas no urbanizadas ofrecen la mayor variedad de sustratos (Cortelezzi, 2010; Ramírez \& Pringle, 1998; Vásquez-Ramos, Guevara-Cardona, \& Reinoso-Flórez, 2014), sin embargo, se desconoce el efecto de microhábitats específicos sobre la riqueza específica y abundancia del ensamble de macroinvertebrados en Guatemala.

Scullion, Parish, Morgan y Edwards (1982) y Logan y Brooker (1983) se delimitaron a comparar áreas deposicionales y rápidos, concluyendo que existe similitud en el número de taxa, sin embargo, las zonas de aguas corrientes presentaron una mayor abundancia y biomasa de macroinvertebrados.

La hojarasca como sustrato para macroinvertebrados acuáticos ha sido uno de los microhábitats más estudiados. Eyes-Escalante, Rodríguez-Barrios y Gutiérrez-Moreno (2012), reportaron 41 taxa y 1,895 individuos en un estudio de descomposición de este material. Crisci, Bispo y Froehlich (2007), confirmaron la alta abundancia y diversidad de organismos en zonas lóticas, enfocando el estudio en los órdenes Ephemeroptera, Trichoptera y Plecóptera. Manrique y Gómez (2017), también estudiaron guijarros, sin embargo, la hojarasca siempre presentó la mayor riqueza. Por último, Cogerino, Cellot y Bournaud (1995), identificaron 18 microhábitats (desde arcilla hasta piedras), de los cuales, la vegetación acuática presentó la mayor riqueza específica.
Se ha evidenciado que la elevada diversidad de insectos acuáticos está asociada a la heterogeneidad del microhábitat disponible, así como también a las condiciones de la calidad del agua que prevalezcan en los cuerpos de agua. Algunos factores medio ambientales pueden llegar a ser más determinantes en la regulación de la ocurrencia y distribución de los insectos acuáticos, como la velocidad de la corriente, temperatura del agua, altitud, estacionalidad, vegetación acuática, disponibilidad de alimento y la competencia interespecífica (Maneechan \& Prommi, 2015).

En Guatemala se realizó un estudio en la ecorregión Lachuá en el cual se quería determinar qué variables físicoquímicas afectaban la distribución de macroinvertebrados a nivel de microhábitat en los ríos Peyán, Lachuá y Tzetoc. Se encontró que la velocidad de corriente y $\mathrm{pH}$ determinaron la distribución en una misma sección de río (García \& Méndez, 2010).

Dentro de la cuenca del lago Atitlán se ha generado información acerca de los macroinvertebrados que se encuentran en los principales ríos. Reyes-Morales y Springer (2014), recolectaron hasta 47,482 individuos de 54 familias y 16 ordenes en 12 sitios de muestreo con distintos usos de suelo. Arriola y Reyes (2016), determinaron que el río Tzununá obtuvo una mayor abundancia de organismos, mientras que el río Panasacar presentó el mayor número de taxa. Hasta la fecha, ninguna investigación se ha dirigido a los microhábitats y el efecto de estos sobre la abundancia y riqueza específica de macroinvertebrados.

El objetivo principal del estudio fue evaluar el efecto que tienen ocho microhábitats (piedras, hojarasca, catarata, roca o peña, pozas, grava, musgo y vegetación acuática) sobre la riqueza específica y abundancia de macroinvertebrados bentónicos de dos ríos tropicales de montaña, dentro de la cuenca del lago Atitlán. También se detalla la composición de dicho ensamble en los microhábitats, demostrando la importancia de algunos sustratos dentro de los ríos. Estos sitios poco intervenidos brindan información valiosa que puede usarse para comparar lo que pasa con estas variables río abajo e incluso en otras cuencas (por ejemplo el río Quiscab), donde se ha reportado una tendencia al deterioro de la calidad biológica (Gil, 2016). Los datos obtenidos también promueven la toma de decisiones en cuanto al manejo adecuado de las cuencas, principalmente a la conservación y/o restauración del hábitat, lo cual fomentaría la heterogeneidad de microhábitats. 


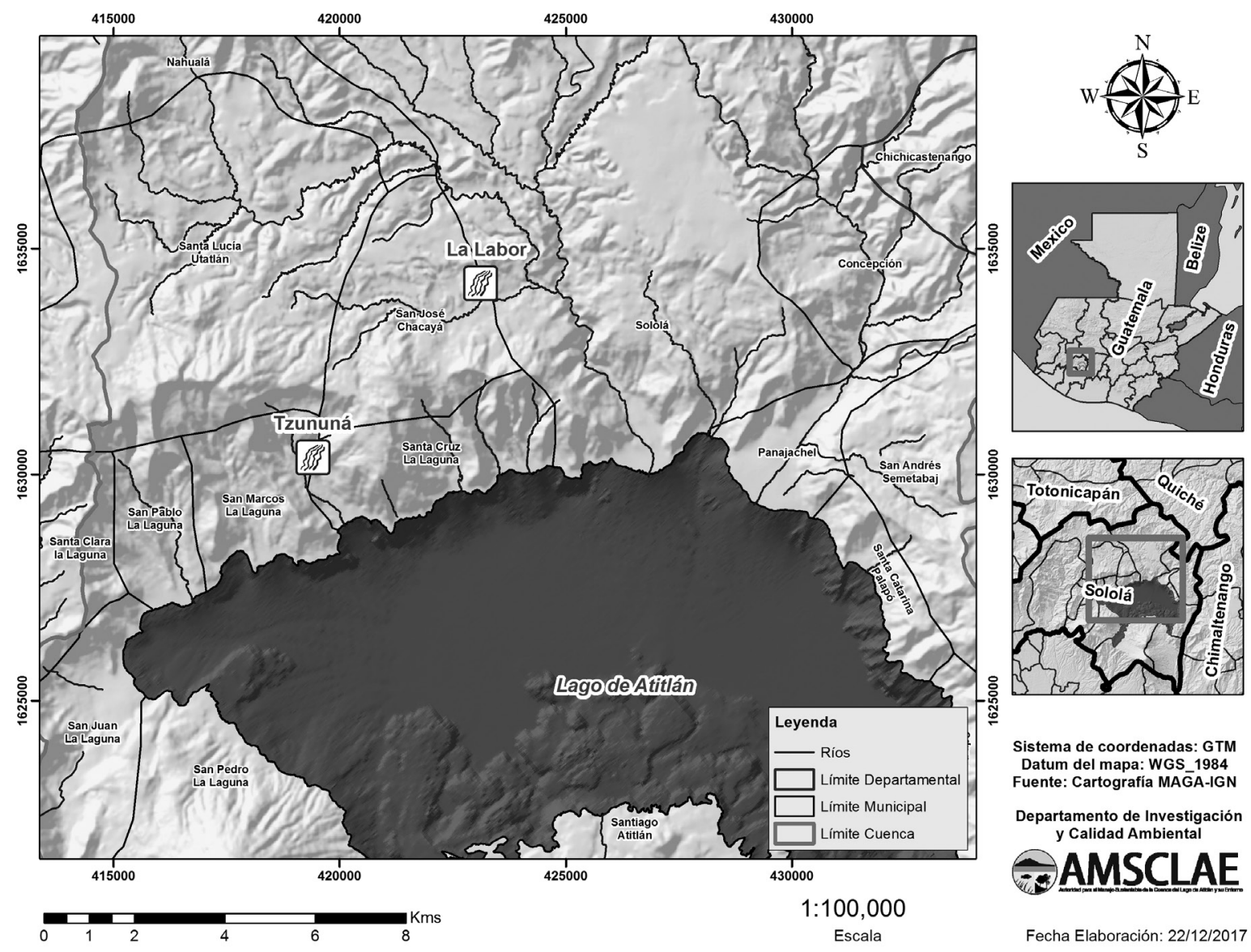

Figura 1. Ubicación geográfica del río La Labor y Tzununá en la cuenca del lago Atitlán, Sololá, Guatemala.

\section{Materiales y métodos}

\section{Área de estudio}

Se seleccionaron dos sitios de muestreo ubicados dentro de la cuenca del lago Atitlán, la cual se encuentra ubicada en el sureste del altiplano de Guatemala (Figura 1).

El río La Labor se ubica en el municipio de San José Chacayá, Sololá, a una altitud de 2,124 msnm. Este río presentó gran cobertura boscosa, caídas de agua de todos tamaños y rocas de tamaño variable. El ancho del río osciló entre los 0.5 y $1.5 \mathrm{~m}$ aproximadamente. Con agua clara e inodora y grandes parches de hojarasca en toda el área. El río Tzununá se ubica en el municipio de Santa Cruz La Laguna, Sololá, a una altitud entre los 1,708 y 1,761 msnm (Arriola \& Reyes, 2016).

Este río presentó áreas boscosas y rocosas en ambos márgenes, una catarata de gran dimensión y un gran número de caídas de agua de tamaño variable. El ancho del río osciló entre los 0.5 y 1.8 m aproximadamente. Con agua clara e inodora y presencia de material rocoso de tamaño variable.

\section{Selección de la muestra}

Se realizó un solo muestreo en cada río en época seca (abril), esto con el fin de evitar perturbaciones en los datos por el efecto de deriva, el cual ocasiona la pérdida de organismos en algunos microhábitats, o bien, 

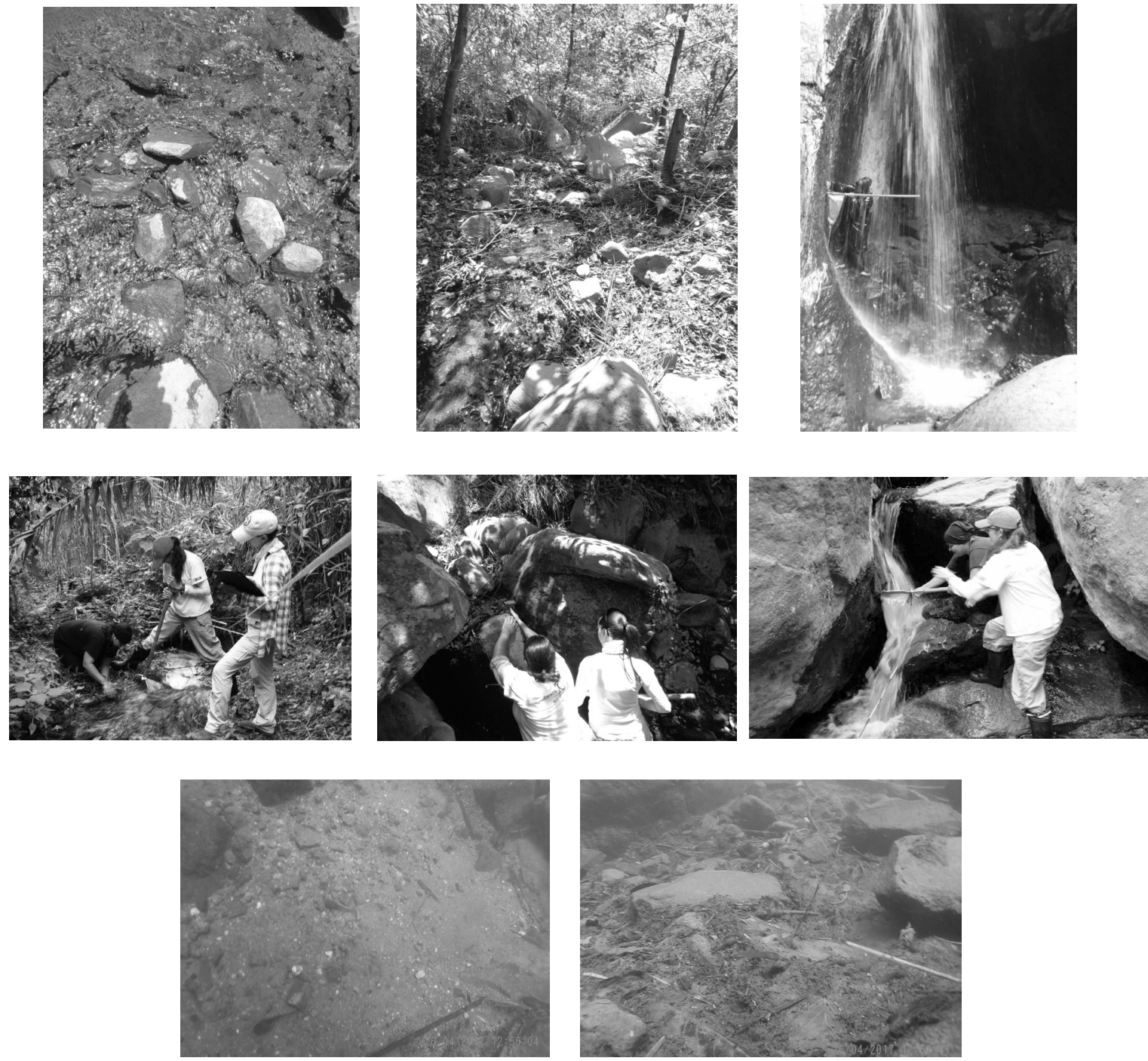

Figura 2. Microhábitats seleccionados. Arriba: piedras o guijarros, hojarasca, musgo. Centro: vegetación acuática, roca o peña, catarata. Abajo: grava o cascajo y pozas.

el arrastre de los organismos de un sitio a otro, lo cual es propio de la temporada lluviosa (Brown \& Brussock, 1991; Reyes-Morales \& Springer, 2014). Se seleccionaron ocho tipos de microhábitats: hojarasca, grava o cascajo, piedras o guijarro, roca o peña, musgo, vegetación acuática, pozas y cataratas (Tabla 1, Figura 2).

Debido a que la investigación es descriptiva, se seleccionó un muestreo no probabilístico, por lo que no se precisó estimar el tamaño de la muestra. Se seleccionaron sitios de poca intervención humana, tomando en cuenta el índice de calidad de hábitat, el cual se realizó por medio del Protocolo de evaluación biológica rápida (RBP) (Barbour, Gerritsen, Snyder, \& Stribling, 1999; Reyes, 2012). La metodología consiste en la evaluación de 10 parámetros físicos del río, los cuales se ponderan de 1 a 20 puntos dependiendo la calidad de la variable evaluada. La sumatoria brinda un número, donde 200 cataloga un sitio como de excelente calidad de hábitat. Los ríos evaluados fueron de buena calidad con puntuaciones de 137 para río La Labor y 156 para río Tzununá, 
Tabla 1

Descripción de los microhábitats

\begin{tabular}{|c|c|}
\hline Micro hábitat & Descripción del microhábitat \\
\hline Hojarasca & $\begin{array}{l}\text { Se encontró en forma de paquetes grandes o dispersos en sitios con corriente que oscilaron } \\
\text { entre los } 0.001 \text { y } 0.382 \mathrm{~m} / \mathrm{s} \text { y profundidades desde } 0.022 \text { a } 0.426 \mathrm{~m} \text {. Los paquetes se encon- } \\
\text { traron en aguas estancadas, pozas y previo a pequeñas caídas de agua. }\end{array}$ \\
\hline Grava o cascajo & $\begin{array}{l}\text { Se encontró en forma de bancos, principalmente dentro de pozas y cerca de piedras o guija- } \\
\text { rro. La velocidad de corriente osciló entre } 0.019 \text { y } 0.215 \mathrm{~m} / \mathrm{s} \text {, mientras que la profundidad } \\
\text { varió entre } 0.010 \text { y } 0 \mathrm{~m} \text {. }\end{array}$ \\
\hline Piedras o guijarro & $\begin{array}{l}\text { Este microhábitat se encontró únicamente en el río Tzununá, donde fue muy abundante y y } \\
\text { ocupaba gran parte del cauce del río, el flujo laminar generalmente era mediano. La veloci- } \\
\text { dad de corriente osciló entre los } 0.014 \text { y } 0.668 \mathrm{~m} / \mathrm{s} \text { y la profundidad entre los } 0.016 \text { y } 0.080 \\
\mathrm{~m} \text {. }\end{array}$ \\
\hline Roca o peña & $\begin{array}{l}\text { Este microhábitat era propio de grandes y pequeñas caías de agua que presentaron un flujo } \\
\text { laminar delgado, la velocidad de corriente osciló entre } 0.017 \text { y } 0.687 \mathrm{~m} / \mathrm{s} \text { y la profundidad } \\
0.005 \text { a } 0.217 \mathrm{~m} \text {. }\end{array}$ \\
\hline Musgo & $\begin{array}{l}\text { Este microhábitat fue abundante únicamente en la zona de salpique de la catarata que pre- } \\
\text { sentó mayor altitud del río Tzununá. La velocidad de corriente varió entre los } 0.001 \text { y } 0.340 \\
\mathrm{~m} / \mathrm{s} \text {, mientras que la profundidad osciló entre los } 0.002 \text { y } 0.011 \mathrm{~m} \text {. }\end{array}$ \\
\hline Vegetación acuática & $\begin{array}{l}\text { Este microhábitat se encontró únicamente en el río Tzununá. La vegetación presente estaba } \\
\text { compuesta principalmente de la familia Commelinaceae y raíces que se encontraron en la } \\
\text { zona ribereña, la cual contaba con plantas de la familia Asteraceae, Poaceae, Urticaceae, } \\
\text { Ulmaceae, Liliaceae y Convolvulaceae. La velocidad de corriente osciló entre los } 0.133 \text { y } \\
0.311 \mathrm{~m} / \mathrm{s} \text {, mientras que la profundidad varió entre } 0.021 \text { y } 0.050 \mathrm{~m} \text {. }\end{array}$ \\
\hline Pozas & $\begin{array}{l}\text { Estos sitios estaban compuestos principalmente de arena, materia orgánica en descomposi- } \\
\text { ción y un poco de hojarasca. La velocidad de corriente varió entre } 0.002 \text { y } 0.246 \mathrm{~m} / \mathrm{s} \text {, y la } \\
\text { profundidad se encontró entre } 0.168 \text { y } 0.383 \mathrm{~m} \text {. }\end{array}$ \\
\hline Catarata & $\begin{array}{l}\text { De este microhábitat se tomó en cuenta la zona de salpique y zona de rápidos. La velocidad } \\
\text { de corriente osciló entre } 0.000 \text { y } 1.604 \mathrm{~m} / \mathrm{s} \text {, y la profundidad entre } 0.000 \text { y } 0.051 \mathrm{~m} \text {. }\end{array}$ \\
\hline
\end{tabular}

la buena calidad se atribuye a la gran heterogeneidad de sustratos disponibles para colonización, poca o nula alteración del cauce, ocurrencia frecuente de rápidos y baja deposición de sedimentos.

Otro criterio para la selección de los sitios de muestreo y la muestra fue la abundancia y riqueza de macroinvertebrados reportada en ambos ríos por Arriola y Reyes (2016) en años anteriores.

\section{Procedimiento}

En cada cuerpo de agua se seleccionó un tramo de aproximadamente $200 \mathrm{~m}$ sobre el cauce principal del río, donde se identificaron todos los microhábitats presentes. En cada microhábitat identificado, se recolectaron muestras de macroinvertebrados con una red en $\mathrm{D}$, por cada muestra se muestreó un área de $900 \mathrm{~cm}^{2}$. Se recolectaron 65 muestras de macroinvertebrados ben- tónicos, de las cuales 23 fueron tomadas en el río La Labor y 42 en el río Tzununá. En cuanto a cantidad de muestras por tipo de microhábitat, se tomaron 12 muestras de hojarasca, grava y piedras, ocho de catarata, siete de pozas, seis de roca y cuatro de musgo y vegetación acuática. Cabe destacar que el esfuerzo de muestreo por cada microhábitat fue distinto, debido a la disponibilidad de cada uno dentro del río. Adicionalmente, se tomaron la velocidad y profundidad en cada punto de muestreo (Tabla 2) por medio de un medidor magnético de caudal $\left(\mathrm{OTT}^{\circledR}\right)$.

Las muestras colectadas se lavaron para retirar el exceso de material fino, se almacenaron en bolsas con cierre hermético y fueron fijadas con alcohol etílico al $95 \%$ e identificadas con su respectiva información para ser trasladadas al laboratorio. En el laboratorio, se procedió a la limpieza de las muestras mediante pinzas entomológicas. Los especímenes se depositaron 
Tabla 2

Promedio y desviación estándar de velocidad y profundidad de muestras de microhábitats

\begin{tabular}{lll}
\hline & Profundidad & Velocidad \\
\hline Catarata & $0.012(0.017)$ & $0.431(0.591)$ \\
Grava & $0.062(0.075)$ & $0.077(0.066)$ \\
Hojarasca & $0.078(0.111)$ & $0.109(0.137)$ \\
Musgo & $0.005(0.004)$ & $0.148(0.174)$ \\
Piedras & $0.041(0.024)$ & $0.313(0.195)$ \\
Poza & $0.209(0.077)$ & $0.045(0.089)$ \\
Roca & $0.091(0.100)$ & $0.337(0.301)$ \\
Vegetación & $0.039(0.013)$ & $0.187(0.083)$ \\
\hline
\end{tabular}

en botes plásticos herméticos y fueron preservados y almacenados en alcohol etílico al 70\%.

Se procedió a la identificación de los organismos hasta el más bajo nivel taxonómico posible, mediante un estereoscopio óptico (Olympus ${ }^{\circledR}$ SZ61) y claves taxonómicas especializadas (Flowers \& De la Rosa, 2010; Gutiérrez-Fonseca, 2010; Merritt, Cummings, \& Berg, 2008; Ramírez, 2010; Silva, Nissimian, \& Ferreira, 2007; Springer, 2010; Vinson, 2006). Cabe destacar que existió limitación en algunos grupos por falta de bibliografía a nivel centroamericano.

\section{Análisis de la información}

La riqueza fue analizada por medio de rarefacción basada en individuos, que es una técnica de interpolación que estima los valores de riqueza de especies en cada microhábitat para diferentes esfuerzos de muestreo, esto permite realizar una comparación objetiva a pesar del tamaño muestral (Kraker-Castañeda \& Cóbar-Carranza, 2011). Este análisis se hizo por medio del programa PAST 2.17c (Hammer et al., 2001).

Por medio del programa Minitab17, se realizó un modelo lineal general (MLG) tomando como variable independiente el microhábitat y las covariables (velocidad y profundidad) para determinar su efecto sobre la abundancia y riqueza de macroinvertebrados (variables dependientes), cada una analizada por separado. Cabe notar que estas últimas dos fueron tratadas aplicando la distribución de Poisson, es decir, una transformación loglinear, la cual aproxima los recuentos obtenidos a la distribución normal. Para comprobar la aplicabilidad del modelo, se comprobó con el análisis de residuos.

\section{Resultados}

\section{Composición del ensamble de macroinverte- brados bentónicos}

Se colectaron 19,529 organismos pertenecientes a 91 taxa, correspondientes a cuatro clases, once órdenes, 49 familias y 65 géneros (Tabla 3). Los taxa más abundantes fueron Tanytarsini (Chironomidae: Diptera) con 3,967 organismos, seguido de Orthocladiinae (Chironomidae: Diptera) con 2,051 organismos y Culoptila (Glossosomatidae: Trichoptera) con 1,536.

Dentro de la composición, se encontró que taxa como Heterelmis, Tipulidae, Americabaetis, Baetis, Calosopsyche y los quironómidos Orthocladiinae, Tanytarsini y Tanypodinae, se encontraron en todos los microhábitats evaluados.

Tabla 3

Abundancia de orden/familia/género por los microhábitats evaluados en los ríos La Labor y Tzununá. Catarata (Cat), grava (Gra),hojarasca (Hoj), musgo (Mus), piedras (Pie), pozas (Poz), roca (Roc), vegetación acuática (Veg)

\begin{tabular}{llllllllll}
\hline ORDEN/Familia & Genero & Cat & Gra & Hoj & Mus & Pie & Poz & Roc & Veg \\
\hline COLEOPTERA & & & & & & & & & \\
Elmidae & Cylloepus & - & - & 17 & 90 & 19 & - & 2 & 3 \\
& Heterelmis & 3 & 52 & 207 & 23 & 141 & 39 & 2 & 91 \\
& Macrelmis & 5 & 12 & 105 & 256 & 119 & 6 & - & 198 \\
& Mycrocylloepus & - & - & 1 & 8 & 2 & - & - & 6
\end{tabular}


Tabla 3

Continuación. Abundancia de orden/familia/género por los microhábitats evaluados en los ríos La Labor y Tzununá. Catarata (Cat), grava (Gra), hojarasca (Hoj), musgo (Mus), piedras (Pie), pozas (Poz), roca (Roc), vegetación acuática (Veg)

\begin{tabular}{|c|c|c|c|c|c|c|c|c|c|}
\hline ORDEN/Familia & Genero & Cat & Gra & Hoj & Mus & Pie & Poz & Roc & Veg \\
\hline & Phanocerus & - & - & 13 & - & 3 & 5 & - & 164 \\
\hline & Gen. Indet. & - & - & - & 193 & - & - & - & 3 \\
\hline Limnichidae & Limnichus & - & - & - & - & - & - & - & 1 \\
\hline Psephenidae & Psephenus & - & - & - & - & - & - & - & 1 \\
\hline Scirtidae & Scirtes & - & 1 & 84 & - & - & - & - & 3 \\
\hline Staphilinidae & & - & - & 7 & 3 & - & - & - & 1 \\
\hline Ptilodactylidae & & - & - & - & - & - & - & - & 1 \\
\hline Hydrophilidae & & - & - & 13 & 4 & 1 & - & - & 7 \\
\hline Dytiscidae & & - & - & 24 & - & - & - & - & - \\
\hline Lampyridae & & 2 & - & - & - & - & - & - & 1 \\
\hline \multicolumn{10}{|l|}{ DIPTERA } \\
\hline \multirow[t]{3}{*}{ Blephaceridae } & Aposonalco & 149 & - & - & 1 & - & - & - & - \\
\hline & Paltostoma & 65 & - & 2 & - & 1 & - & 1 & - \\
\hline & Gen. Indet & 1 & - & - & - & - & - & - & - \\
\hline \multirow[t]{4}{*}{ Ceratopogonidae } & Atrichopogon & 1 & - & 2 & - & 1 & - & - & 3 \\
\hline & Bezzia & 3 & 16 & 36 & 19 & 10 & 30 & - & - \\
\hline & Probezzia & - & - & 1 & - & - & 1 & - & 1 \\
\hline & Gen. Indet. & - & 2 & 1 & 1 & - & 1 & - & - \\
\hline \multirow[t]{2}{*}{ Simuliidae } & Simulium & 92 & 2 & 54 & 17 & 38 & - & 499 & 94 \\
\hline & Gen. Indet. & - & - & - & 2 & 1 & - & - & - \\
\hline Dixidae & Dixella & - & 1 & 4 & 1 & 4 & 1 & - & 9 \\
\hline \multirow[t]{3}{*}{ Empididae } & Clinocera & - & - & 14 & - & 3 & - & - & 2 \\
\hline & Neoplasta & - & - & 9 & - & - & - & - & - \\
\hline & Gen. Indet. & - & 1 & 2 & - & - & - & - & - \\
\hline \multirow[t]{3}{*}{ Psychodidae } & Maruina & 450 & - & - & 12 & 6 & - & 2 & - \\
\hline & Psychoda & - & - & 1 & 1 & 10 & - & 2 & 2 \\
\hline & Gen. Indet. & 5 & - & - & 4 & 12 & - & - & - \\
\hline Chironomidae & & 1 & 19 & 89 & 11 & 46 & 11 & 3 & - \\
\hline Chironominii & & - & 56 & 211 & 2 & 14 & 47 & - & 2 \\
\hline Orthocladiinae & & 40 & 44 & 848 & 856 & 139 & 27 & 51 & 46 \\
\hline Tanypodinae & & 2 & 41 & 566 & 224 & 14 & 33 & 1 & 1 \\
\hline Tanytarsini & & 51 & 300 & 2,143 & 856 & 491 & 106 & 14 & 6 \\
\hline Dolichopodidae & & - & - & - & - & - & 1 & - & - \\
\hline Ephydridae & & - & - & 1 & - & - & - & 1 & - \\
\hline
\end{tabular}


Tabla 3

Continuación. Abundancia de orden/familia/género por los microhábitats evaluados en los ríos La Labor y Tzununá. Catarata (Cat), grava (Gra),hojarasca (Hoj), musgo (Mus), piedras (Pie), pozas (Poz), roca (Roc), vegetación acuática (Veg)

\begin{tabular}{|c|c|c|c|c|c|c|c|c|c|}
\hline ORDEN/Familia & Genero & Cat & Gra & Hoj & Mus & Pie & $\mathrm{Poz}$ & Roc & Veg \\
\hline Muscidae & & 1 & - & 1 & 40 & - & 1 & - & - \\
\hline Stratiomyidae & & 3 & - & 1 & 1 & 1 & 2 & - & 1 \\
\hline Tabanidae & & - & - & - & 1 & - & - & - & - \\
\hline Tipulidae & & 2 & 24 & 56 & 9 & 1 & 15 & 1 & 6 \\
\hline \multicolumn{10}{|c|}{ EPHEMEROPTERA } \\
\hline \multirow[t]{7}{*}{ Baetidae } & Americabaetis & 12 & 227 & 625 & 89 & 159 & 31 & 6 & 15 \\
\hline & Baetis & 11 & 18 & 69 & 11 & 195 & 2 & 48 & 9 \\
\hline & Baetodes & 52 & - & 6 & 1 & 23 & - & 102 & 1 \\
\hline & Camelobaetidius & - & 7 & 6 & - & 45 & 2 & 4 & - \\
\hline & Cloeodes & - & 1 & 1 & - & - & - & - & - \\
\hline & Fallceon & 1 & 232 & 1 & - & 10 & 21 & 2 & - \\
\hline & Gen. Indet. & 4 & 43 & 58 & 20 & 30 & 22 & 17 & 9 \\
\hline Heptageniidae & Epeorus & 1 & - & - & - & 28 & - & - & - \\
\hline \multirow[t]{2}{*}{ Leptohyphidae } & Leptohyphes & - & 15 & 92 & - & 75 & - & - & 193 \\
\hline & Tricorythodes & - & 1 & 9 & - & - & 1 & - & - \\
\hline Leptophlebiidae & Farrodes & 1 & 59 & 1,201 & - & 28 & 190 & 2 & 32 \\
\hline \multicolumn{10}{|l|}{ HEMIPTERA } \\
\hline Belostomatidae & Abedus & - & - & 1 & - & - & - & - & 3 \\
\hline Gerridae & Aquarius? & 1 & 1 & - & - & 15 & 3 & 3 & - \\
\hline Hebridae & Hebrus & - & - & - & - & - & - & - & 1 \\
\hline Naucoridae & Cryphocricos & 19 & - & 2 & - & 2 & - & 1 & 1 \\
\hline Notonectidae & Notonecta & - & - & - & - & - & 1 & - & - \\
\hline \multirow[t]{3}{*}{ Veliidae } & Microvelia & 1 & 3 & 4 & 4 & 2 & - & 1 & - \\
\hline & Rhagovelia & - & 5 & 3 & - & - & 7 & - & 5 \\
\hline & Gen. Indet. & - & 8 & 10 & - & 1 & - & 1 & - \\
\hline \multicolumn{10}{|l|}{ LEPIDOPTERA } \\
\hline Crambidae & Petrophila & 3 & - & 1 & - & 4 & - & - & 2 \\
\hline \multicolumn{10}{|l|}{ ODONATA } \\
\hline Aeshnidae & Rhionaeschna? & - & - & 1 & - & 3 & - & - & - \\
\hline Calopterygidae & Hetaerina & - & 2 & 14 & - & - & 1 & - & 21 \\
\hline Coenagrionidae & Argia & - & - & 43 & - & - & - & - & - \\
\hline Gomphidae & Epigomphus & - & - & - & - & 1 & - & - & - \\
\hline Libellulidae & & - & 5 & 2 & - & 5 & - & - & - \\
\hline
\end{tabular}


Tabla 3

Continuación. Abundancia de orden/familia/género por los microhábitats evaluados en los ríos La Labor y Tzununá. Catarata (Cat), grava (Gra), hojarasca (Hoj), musgo (Mus), piedras (Pie), pozas (Poz), roca (Roc), vegetación acuática (Veg)

\begin{tabular}{|c|c|c|c|c|c|c|c|c|c|}
\hline ORDEN/Familia & Genero & Cat & Gra & Hoj & Mus & Pie & $\mathrm{Poz}$ & Roc & Veg \\
\hline \multicolumn{10}{|l|}{ PLECOPTERA } \\
\hline Perlidae & Anacroneuria & - & - & 5 & - & 39 & 1 & - & 7 \\
\hline \multicolumn{10}{|l|}{ TRICHOPTERA } \\
\hline \multirow[t]{2}{*}{ Glossosomatidae } & Culoptila & 4 & 9 & 147 & - & 1,085 & 2 & 247 & 42 \\
\hline & Gen. Indet. & - & - & - & - & 13 & - & 1 & - \\
\hline Helicopsychidae & Helicopsyche & - & 1 & 19 & - & 40 & 2 & 15 & 12 \\
\hline Hydrobiosidae & Atopsyche & 8 & 2 & 27 & 23 & 51 & - & 2 & 1 \\
\hline \multirow{3}{*}{ Hydropsychidae } & Calosopsyche & 2 & 35 & 222 & 56 & 199 & 1 & 15 & 227 \\
\hline & Leptonema & - & - & - & - & 3 & - & - & 6 \\
\hline & Gen. Indet. & 4 & 1 & 24 & - & 8 & 2 & - & 1 \\
\hline \multirow[t]{5}{*}{ Hydroptilidae } & Cerasmotrhichia & 3 & - & - & 26 & - & - & 2 & - \\
\hline & Hydroptila & 17 & 2 & 3 & 99 & 6 & - & 20 & - \\
\hline & Leucotrichia & 1 & - & - & - & - & - & - & - \\
\hline & Mayatrichia & - & 1 & 2 & - & 5 & - & 2 & 3 \\
\hline & Metrichia & 1 & - & - & - & 5 & - & 1 & - \\
\hline \multirow[t]{2}{*}{ Lepidostomatidae } & Lepidostoma & - & 4 & 235 & - & 16 & 2 & 5 & 178 \\
\hline & Gen. Indet. & - & - & 56 & - & - & - & - & - \\
\hline \multirow[t]{3}{*}{ Leptoceridae } & Nectopsyche & - & 1 & 2 & - & - & - & - & - \\
\hline & Oecetis & - & - & 1 & - & - & - & - & - \\
\hline & Triaenodes & - & - & - & - & - & - & - & 1 \\
\hline Philopotamidae & Wormaldia & 6 & - & 9 & 66 & 5 & - & 1 & - \\
\hline \multirow[t]{2}{*}{ Polycentropodidae } & Cernotina & - & 5 & 253 & - & 18 & 30 & 2 & - \\
\hline & Cyrnellus & 1 & - & - & - & - & - & - & - \\
\hline \multicolumn{10}{|l|}{ NEOOPHORA } \\
\hline Dugesiidae & & 1 & 2 & 54 & 16 & 40 & - & - & 45 \\
\hline TROMBIDIFORMES & & 1 & - & - & 2 & - & - & - & - \\
\hline TUBIFICIDA & & 5 & 13 & - & 6 & 2 & 5 & - & 3 \\
\hline COLLEMBOLA & & - & - & 2 & 2 & - & - & - & - \\
\hline Abundancia total & & 1,036 & 1,274 & 7,723 & 3,056 & 3,238 & 652 & 1,079 & 1,471 \\
\hline Número de taxa & & 42 & 41 & 65 & 39 & 56 & 35 & 35 & 49 \\
\hline
\end{tabular}


También se registraron taxa exclusivos de ciertos microhábitats, por ejemplo, en hojarasca se encontró Neoplasta, Argia, Oecetis y Dytiscidae; en vegetación se registró la presencia de Psephenus, Ptilodactylidae, Hebrus y Triaenodes; Tabanidae se encontró únicamente en musgos, Epigomphus en piedras, por último, Notonecta y Dolichopodidae en pozas.

\section{Riqueza especifica de macroinvertebrados ben- tónicos por microhábitat}

La curva de rarefacción permitió hacer una comparación objetiva de la riqueza de especies para una muestra estándar de 641 individuos (Figura 3), donde se obtuvieron los siguientes valores: piedras (41), vegetación (38), hojarasca (37), catarata (35), pozas (34), grava (34), roca o peña (29) y musgo (28).

\section{Abundancia de macroinvertebrados bentónicos por microhábitat}

En cuanto a las abundancias por microhábitat destacó hojarasca $(7,723$ organismos $)$, piedras $(3,238)$ y musgo $(3,056)$, se encontraron abundancias relativamente grandes en vegetación $(1,471)$, grava $(1,274)$, roca o peña $(1,079)$ y cataratas $(1,036)$, por último, la menor abundancia se encontró en pozas (652).

En hojarasca, se registró mayor abundancia de los taxa Tanytarsini $(2,143)$, Farrodes $(1,201)$ y Orthocladiinae (848). En piedras predominó Culoptila (1,085), Tanytarsini (491) y Calosopsyche (199). En musgo Tanytarsini (856), Orthocladiinae (856) y $M a-$ crelmis (256), en vegetación dominó Calosopsyche (227), Macrelmis (198) y Leptohyphes (193), mientras que en grava abundó Tanytarsini (300), Fallceon (232) y Americabaetis (227), en roca o peña Simulium (499), Culoptila (247) y Baetodes (102), en cataratas dominó Maruina (450), Aposonalco (149) y Simulium (92) y en pozas Farrodes (190), Tanytarsini (106) y Chironominii (47).

\section{Efecto de microhábitats y variables ambientales sobre la riqueza específica y abundancia}

El análisis de varianza del modelo lineal general indicó que los microhábitats catarata $(p=.001)$, hojarasca $(p>.001)$, piedras $(p=.001)$ y roca $(p=.002)$ tienen un efecto significativo en la riqueza de taxo- nes de macroinvertebrados bentónicos, al igual que la profundidad ( $p=.030)$, la cual presentó una relación inversa. La velocidad no mostró efecto alguno.

El análisis de varianza del modelo linear general mostró que el microhábitat tiene un efecto significativo en la abundancia de macroinvertebrados, específicamente grava $(p=.007)$ y hojarasca $(p>.001)$, mientras que las variables ambientales, velocidad de corriente $(p=.864)$ y profundidad $(p=.085)$, no presentaron significancia con respecto a la abundancia.

\section{Discusión}

La presencia de Heterelmis, Tipulidae, Americabaetis, Baetis, Calosopsyche y los quironómidos Orthocladiinae, Tanytarsini y Tanypodinae en todos los microhábitats pone en evidencia que los mismos taxa pueden encontrarse en una amplia gama de velocidades y profundidades, debido al microambiente complejo y la capacidad de tolerancia de los organismos (Brown \& Brussock, 1991; Jowett, 2003).

La poca abundancia de organismos en pozas puede deberse a la baja diversidad de sustratos, los cuales reducen la disponibilidad de espacios intersticiales, el bajo suministro de alimento y de oxígeno (Sepulcre, 2012). La riqueza fue relativamente alta, principalmente por el orden Diptera, quienes son afines a este hábitat (Moya, Gibon, Oberdorff, Rosales, \& Domínguez, 2009). Aunque pozas y grava presentaron la misma riqueza taxonómica y un hábitat pobre, este último microhábitat sostuvo una mayor abundancia de organismos colectores como Fallceon y Americabaetis (Ephemeroptera) y quironómidos, quienes aprovechan la cantidad de detritus y arena acumulada (Chacón, 2003; Rodríguez-Barrios, Ospina-Torres, \& Turizo-Correa, 2011; Vásquez-Ramos \& Reinoso-Flórez, 2012).

Cataratas fue un microhábitat singular, la alta presencia de organismos raspadores como Maruina (Psychodidae) y Aposonalco (Blephaceridae), así como el filtrador Simulium (Simuliidae), presentan adaptaciones morfológicas especializadas como la obtención de oxígeno por la piel, estructuras de fijación como ventosas y producción de seda, las cuales permitieron su establecimiento en microhábitats expuestos a corrientes (Merritt et al., 2008).

El efecto abrasivo de la corriente sobre el sustrato limita a grupos como Trichoptera y Coleoptera, ya que disminuye la disponibilidad y estabilidad del hábitat (Vásquez-Ramos \& Reinoso-Flórez, 2012). Este caso 
fue evidente en catarata donde predominó Diptera, sin embargo, roca o peña, que presentó velocidad de corriente menor, posibilitó el establecimiento y abundancia de Trichoptera (Culoptila), Baetodes (Ephemeroptera) y Simulium (Diptera), pero no de Coleoptera.

Piedras o guijarro fue uno de los microhábitats con más riqueza y abundancia de macroinvertebrados. El tipo de sustrato es idóneo para el crecimiento de algas y la disponibilidad de espacios intersticiales que permiten la presencia de materia orgánica, ambas fuentes importantes de alimento (Velásquez \& Miserendino, 2003). Así mismo, este microhábitat brinda refugio principalmente a Culoptila y Calosopsyche (Trichoptera), los cuales se encontraron en abundancias importantes.

Según Dodds (2002), "las aguas rápidas deberían ser más ricas en nutrientes, lo que permitiría la presencia de especies filtradoras" (citado en Vásquez-Ramos \& Reinoso-Flórez, 2012, p. 356), esto explica la alta presencia de Simulium en cataratas y roca, así como de Calosopsyche en piedras. Estos tres microhábitats presentaron corriente media a alta y según el modelo lineal general, ejercen un efecto sobre la riqueza, la cual fue bastante alta en piedras, media en cataratas y pequeña en roca o peña, por lo que la relación puede deberse a la textura del sustrato.

El material vegetal se considera importante debido a que brinda estructura física, así como alimento abundante y de buena calidad. Estos factores influenciaron positivamente la riqueza en microhábitats como la vegetación acuática (Meza, Rubio, Días, \& Walteros, 2012; Rivera, 2004).

La hojarasca se encuentra fácilmente en tramos boscosos poco intervenidos y brinda una mayor disponibilidad de recursos (Burdett \& Watts, 2009; Meza et al., 2012), por lo que alberga la mayor abundancia y riqueza de taxa de macroinvertebrados, tanto en zona de rápidos como de remansos (Baptista, Buss, Dorvillé, \& Nessimian, 2001).

La alta heterogeneidad de hábitat de la hojarasca ofrece buena fuente de alimento, lo cual promueve la alta abundancia de organismos colectores como Tanytarsini y Orthocladiinae (Chironomidae) (Rodríguez-Barrios et al., 2011). Según Chará-Serna y colaboradores (2012), la gran abundancia de Farrodes se debe a la degradación de las hojas las cuales brindan materia orgánica fina, el principal alimento de este taxa (citado en Sajamí \& Huamantico, 2016, p. 100).

Estos atributos definen la importancia de los bosques en los sistemas lóticos. Se ha demostrado que la disminución en la cobertura boscosa afecta la calidad de hábitat de los ríos, ya que genera aumento en la longitud del cauce y temperatura del agua, fomenta la sedimentación en zonas de rápidos y promueve la cobertura perifítica, por lo que afecta significativamente la abundancia de macroinvertebrados (Davies \& Nelson, 1994). La hojarasca es un microhábitat importante en la comunidad de macroinvertebrados, ya que posee un efecto sobre la abundancia, además en sistemas tropicales, los paquetes de hojas se encuentran todo el año (Ramírez, Paaby, Pringle, \& Agüero, 1998).

Se ha registrado que la alteración de las condiciones ecológicas dentro de los ríos afecta a los grupos ya establecidos en cuanto a abundancia. Por lo que promueve la colonización de otros grupos, los cuales también afectan la distribución de la comunidad de macroinvertebrados dentro de los sistemas acuáticos (Reyes, 2012). Esto demuestra la importancia del buen manejo de las cuencas, en especial la del lago Atitlán. Donde existen amenazas como la construcción de nuevas vías de acceso, expansión de la frontera agrícola, urbanización, incendios forestales, degradación de hábitats y contaminación (Rejmánková et al., 2018). Dichas acciones afectan negativamente la calidad de hábitat de los ríos, por ende, la disponibilidad de microhábitats y la estructura de la comunidad de macroinvertebrados.

Como se ha comprobado, el microhábitat juega un papel fundamental en la riqueza de macroinvertebrados. Algunos autores confirman los resultados obtenidos en esta investigación, incluso han reportado que ciertos parámetros fisicoquímicos pueden influenciar la composición de macroinvertebrados, tales como sólidos disueltos, pH, temperatura y turbidez (Moya et al., 2009; Roy \& Homechaudhuri, 2017).

Esta investigación demostró que el microhábitat es muy importante para determinar la composición de la comunidad de macroinvertebrados (Costa \& Sánchez, 2008). La presencia y dominancia de elementos de heterogeneidad en los ríos contribuye a incrementar la diversidad de hábitat físico y de las fuentes alimenticias como material alóctono y autóctono, además de proveer refugio en abundancia (Pardo et al., 2002; Villamarín, 2008), esto determina la riqueza y productividad de los macroinvertebrados en los ríos (Velásquez \& Miserendino, 2003).

Por último, la información generada debe permitir la toma de decisiones que mejoren las condiciones ecológicas de los ríos de la cuenca del lago Atitlán. Primordialmente se debe mantener la heterogeneidad de hábitats en los cuerpos de agua, los cuales a su vez 


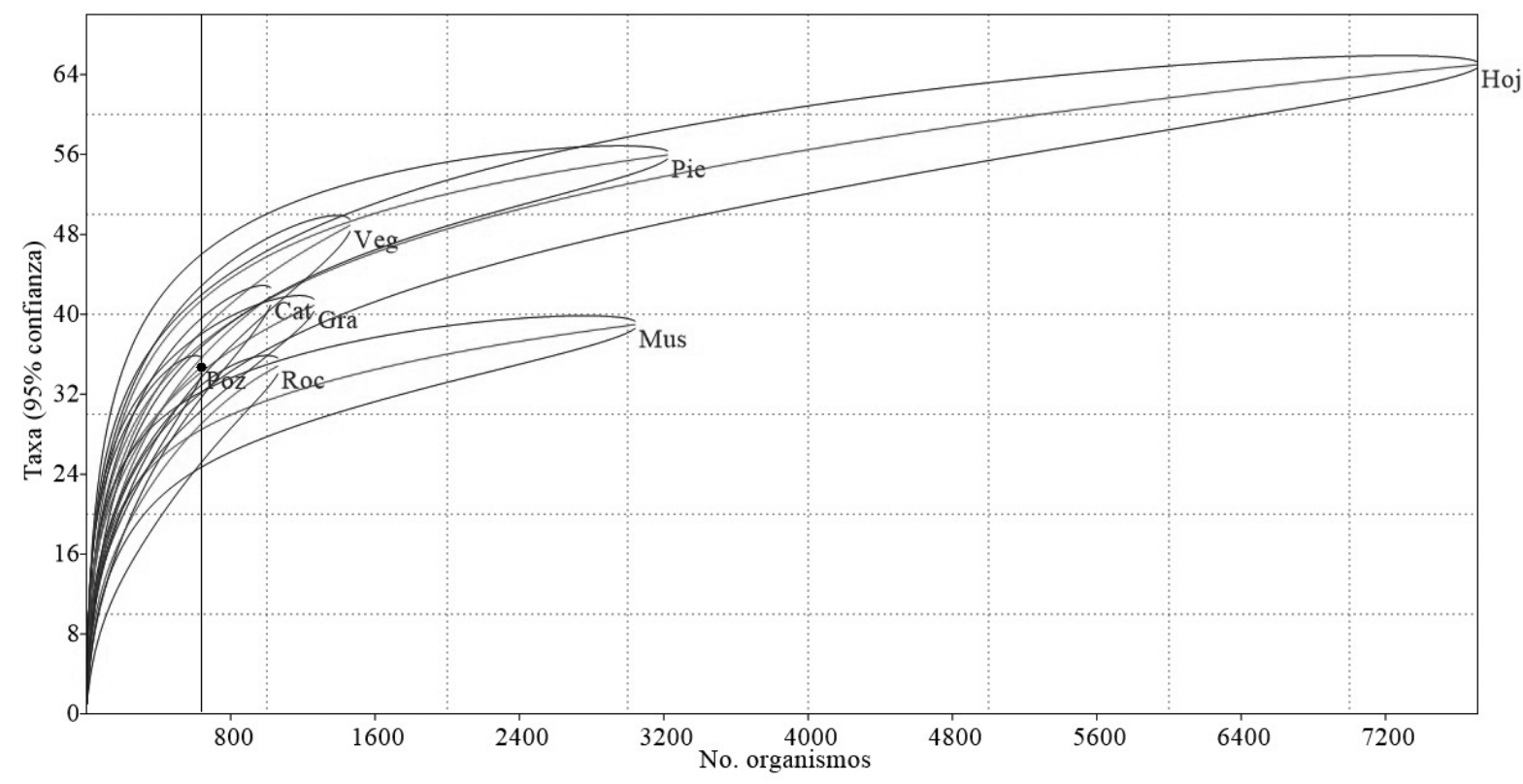

Figura 3. Curva de rarefacción para una muestra estándar de 641 individuos. Línea roja: curva de rarefacción y línea azul: intervalo de confianza $95 \%$.

mantienen la población de macroinvertebrados. Esto se alcanza a través del buen manejo de la cuenca, principalmente la restauración de bosques nativos, los cuales aumentan microhábitats como hojarasca (uno de los más productivos). El buen manejo también puede alcanzarse por medio de la educación ambiental, sobre todo en la importancia de la zona ribereña de, ya que existe gran expansión de la frontera agrícola y de urbanización, las cuales fomentan la degradación de hábitats y contaminación.

\section{Agradecimientos}

La realización de este trabajo ha sido posible gracias a la colaboración de Isabel Arriola, Cristina Martínez, Federico Nave, Natanael Xamínez, Domingo Ujpán, Flor Barreno y Cristian Craker.

\section{Referencias}

Alonso, A., \& Camargo, J. A. (2005). Estado actual y perspectivas en el empleo de la comunidad de macroinvertebrados bentónicos como indicadora del estado ecológico de los ecosistemas fluviales españoles. Ecosistemas, 14(3), 87-99. doi:10.7818/ re.2014.14-3.00.
Arriola, A. I., \& Reyes, E. M. F. (2016). Informe de calidad de agua de ríos de la cuenca del lago de Atitlán 2015. Panajachel, Sololá, Guatemala: Autoridad para el Manejo Sustentable de la Cuenca del Lago de Atitlán y su Entorno Departamento de Investigación y Calidad Ambiental. Recuperado de http://www.amsclae.gob.gt/wp-content/ uploads/2016/12/rios2016.pdf

Baptista, D. F., Buss, D. F., Dorvillé, L. F. M., \& Nessimian, J. L. (2001). Diversity and habitat preference of aquatic insects along the longitudinal gradient of the macaé river basin, Rio de Janeiro, Brazil. Revista Brasileira de Biología, 61(2), 249258. doi:10.1590/S0034-71082001000200007

Barbour, M. T., Gerritsen, J., Snyder, B. D., \& Stribling, J. B. (1999). Rapid bioassessment protocols for use in streams and wadeable rivers: Periphyton, benthic macroinvertebrates and Fish ( $2^{\text {nd }}$ ed.). Washington DC: U.S. Environmental Protection Agency.

Brown, A. V., \& Brussock, P. P. (1991). Comparisons of benthic invertebrates between riffles and pools. Hydrobiologia, 220(2), 99-108. doi: 10.1007/ BF00006542. 
Burdett, A. S., \& Watts, R. J. (2009). Modifying living space: An experimental study of the influences of vegetation on aquatic invertebrate community structure. Hydrobiologia, 618(1), 161-173. doi:10.1007/s10750-008-9573-z

Chacón, M. M. (2003). Comunidades de Ephemeroptera (Insecta) en la cuenca del rio Chama y su relación con la variabilidad ambiental (Tesis de doctorado). Universidad de los Andes, Mérida.

Cogerino, L., Cellot, B., \& Bournaud, M. (1995). Microhabitat diversity and associated macroinvertebrates in aquatic banks of a large European river. Hydrobiologia, 304(2), 103-115. doi:10.1007/ BF02579416

Cortelezzi, A. (2010). Hábitats funcionales y macroinvertebrados en cauces modificados de arroyos de llanura (Tesis de doctorado). Universidad Nacional de La Plata, La Plata.

Costa, S. S., \& Sánches, A. (2008). Beta diversity in stream macroinvertebrate assemblages: among-site and among-microhabitat components. Hydrobiologia, 598(1), 131-138 doi:10.1007/s10750007-9145-7

Covich, A. P., Palmer, M. A., \& Crowl, T. A. (1999). The role of benthic invertebrate species in freshwater ecosystems. BioScience, 49(2), 119-127.

Crisci, V., Bispo, P., \& Froehlich, C. (2007). Ephemeroptera, Plecoptera and Trichoptera assemblages in litter in a mountain stream of Atlantic Rainforest from Southeastern Brazil. Revista Brasileira de Zoologia, 24(3), 545-551. doi:10.1590/S010181752007000300004

Davies, P. E., \& Nelson, M. (1994). Relationships between riparian buffer widths and the effects of logging on stream habitat, invertebrate community composition and fish abundance. Australian Journal of Marine and Freshwater Research, 45(7), 1289-1305.

Eyes-Escalante, M., Rodríguez-Barrios, J., \& Gutiérrez-Moreno, L. C. (2012). Descomposición de la hojarasca y su relación con los macroinvertebrados acuáticos del río Gaira (Santa Marta-Colombia). Acta Biológica Colombiana, 17(1), 77-92.

Flowers, R. W., \& De la Rosa, C. (2010). Ephemeroptera. Revista Biología Tropical, 58(4), 63-93.

García, P., \& Méndez, C. (2010). Análisis de la distribución de macroinvertebrados acuáticos a escala detallada en la ecorregión Lachuá, Cobán, Alta Verapaz. Revista Cientifica del Instituto de Investigaciones Químicas y Biológicas, 19(2), 37-50.

Gil, N. E. (2016). Caracterización y evaluación de la calidad del agua de los ríos Quiscab y San Francisco, Guatemala. Ciencia, Tecnología y Salud, 3(2), 193.

Gutiérrez-Fonseca, P. E. (2010). Guía ilustrada para el estudio ecológico y taxonómico de los insectos acuáticos del Orden Coleoptera en El Salvador. En M Springer \& J. M. Sermeño (Eds.), Formulación de una guía metodológica estandarizada para determinar la calidad ambiental de las aguas de los ríos de El Salvador, utilizando insectos acuáticos. San Salvador, El Salvador: Editorial Universitaria (UES).

Hammer, Ø. (Ed.) (2001). Paleontological statistics. Reference Manual. Noruega: Natural History Museum. University of Oslo,

Jiménez-Sandoval, J. A. (2015). Morfología funcional $y$ hábitat de macroinvertebrados acuáticos en lagunas del parque nacional natural Chingaza (Tesis de licenciatura). Pontificia Universidad Javeriana, Bogotá.

Jowett, I. G. (2003). Hydraulic constraints on habitat suitability for benthic invertebrates in grave-bed rivers. River Research and Applications, 19(6), 495-507. doi:10.1002/rra.734

Kraker-Castañeda, C., \& Cóbar-Carranza, A. J. (2011). Uso de rarefacción para comparación de la riqueza de especies: el caso de las aves de sotobosque en la zona de influencia del Parque Nacional Laguna Lachuá, Guatemala. Naturaleza y Desarrollo, 9(1), 60-70.

Logan, P., \& Brooker, M. P. (1983). The macroinvertebrate faunas of rifles and pools. Water Research, 17(3), 263-270. doi:10.1016/00431354(83)90179-3.

Maneechan, W., \& Prommi, T. O. (2015). Diversity and distribution of aquatic insects in streams of the Mae Klong watershed, western Thailand. Psyche, 2015(1), 1-7. doi:10.1155/2015/912451.

Manrique, E., \& Gómez, S. (2017). Colonización de sustratos por macroinvertebrados acuáticos en las aguas de la quebrada Charquira del municipio de Carmen de Carupa (Tesis de licenciatura). 
Universidad Distrital Francisco José de Caldas, Bogotá.

Merritt, R. W., Cummins, K. W., \& Berg, M. B. (Eds.). (2008). An introduction to the aquatic insects of North America. Dubuque, Iowa: Kendall/Hunt Publishing.

Meza, A. M., Rubio, J., Dias, L. G., \& Walteros, J. M. (2012). Calidad de agua y composición de macroinvertebrados acuáticos en la subcuenca alta del río Chinchiná. Caldasia, 34(2), 443-456.

Moya, N., Gibon, F. M., Oberdorff, T., Rosales, C., \& Domínguez, E. (2009). Comparación de las comunidades de macroinvertebrados acuáticos en ríos intermitentes y permanentes del altiplano boliviano: Implicaciones para el futuro cambio climático. Ecología Aplicada, 8(2), 105-114.

Pardo, I., Álvarez, M., Casas, J., Moreno, J. L., Vivas, S., Bonada, N., ... Vidal-Abarca, M. R. (2002). El hábitat de los ríos mediterráneos: Diseño de un índice de diversidad de hábitat. Limnetica, 21(34), 115-133.

Ramírez, A. (2010). Odonata. Revista Biología Tropical, 58(4), 97-136.

Ramírez, A., Paaby, P., Pringle, C. M., \& Agüero, G. (1998). Effect of habitat type on benthic macroinvertebrates in two lowland tropical streams, Costa Rica. Revista Biología Tropical, 46(6), 201-213. doi:10.15517/rbt.v46i6.29826

Ramírez, A., \& Pringle, C. M. (1998). Structure and production of a benthic insect assemblage in a neotropical stream. Journal of the North American Benthological Society, 14(4), 443-463. doi:10.2307/1468365

Rejmánková, E., Sullivan, B. W., Ortiz, J. R., Snyder, J. M., Castle, S. T., \& Reyes, F. (2018). Regime shift in the littoral ecosystem of volcanic Lake Atitlán in Central America: combined role of stochastic event and invasive plant species. Freshwater Biology, 1-19. doi:10.1111/fwb.13119.

Reyes, E. M. F. (2012). Uso de macroinvertebrados acuáticos como indicadores biológicos de la calidad del agua en la cuenca del lago Atitlán, Guatemala (Tesis de maestría). Universidad de Costa Rica, San José, Costa Rica.

Reyes-Morales, F., \& Springer, M. (2014). Efecto del esfuerzo de muestreo en la riqueza de taxones de macroinvertebrados acuáticos y el índice BMWP/ Atitlán. Revista Biología Tropical, 62(2), 291301.

Rivera, R. (2004). Estructura y composición de la comunidad de macroinvertebrados bentónicos en ríos de páramo y zonas boscosas, en los andes venezolanos (Tesis de licenciatura). Universidad de los Andes, Mérida, Venezuela.

Rodríguez-Barrios, J., Ospina-Torres, R., \& Turizo-Correa, R. (2011). Grupos funcionales alimentarios de macroinvertebrados acuáticos en el río Gaira, Colombia. Revista de Biología Tropical, 59(4), 1537-1552. doi: 10.15517/rbt.v59i4.3418

Roy, A., \& Homechaudhuri, S. (2017). Comparing diversity of freshwater macroinvertebrate community along habitat gradients within a riverine system in North Bengal, India. Journal of Entomology and Zoology Studies, 5(4), 86-93.

Sajamí, J. I., \& Huamantico, A. A. (2016). Distribución espacial de Ephemeroptera, Plecoptera, Trichoptera y Coleoptera (Insecta) en una quebrada de primer orden, bosque montano, Junín, Perú. Revista Peruana de Biología, 23(2), 95-102. doi:10.15381/rpb.v23i2.12377

Scullion, J., Parish, C. A., Morgan, N., \& Edwards, R. W. (1982). Comparison of benthic macroinvertebrate fauna and substratum composition in riffles and pools in the impounded river Elan and de unregulated river Wye, mid-wales. Freshwater Biology, 12(6), 579-595. doi:10.1111/j.1365-2427.1982. tb00650.x

Sepulcre, N. (2012). Estudio de los macroinvertebrados acuáticos en ríos europeos de montaña en relación con las características del microhábitat (Tesis de licenciatura). Universidad Politécnica de Valencia, Escuela Politécnica Superior de Gandia, Valencia.

Silva, M. I., Nessimian, J. L., \& Ferreira, N. (2007). Chaves para identificação dos gêneros de Elmidae (Coleoptera) ocorrentes no Estado do Rio de Janeiro, Brasil. Revista Brasileira de Entomologia, 51(1), 42-53. doi:10.1590/S008556262007000100008

Springer, M. (2010). Trichoptera. Revista Biología Tropical, 58(4), 151-198. 
Vásquez-Ramos, J. M., Guevara-Cardona, G., \& Reinoso-Flórez, G. (2014). Factores ambientales asociados con la preferencia de hábitat de larvas de tricópteros en cuencas con bosque seco tropical (Tolima, Colombia). Revista Biología Tropical, 62(2), 21-40.

Vásquez-Ramos, J. M., \& Reinoso-Flórez, G. (2012). Estructura de la fauna béntica en corrientes de los Andes colombianos. Revista Colombiana de Entomología 38(2), 351-358.

Velásquez, S. M., \& Miserendino, M. L. (2003). Análisis de la materia orgánica alóctona y organización funcional de macroinvertebrados en relación con el tipo de hábitat en ríos de montaña de Patagonia. Ecología Austral, 13, 67-82.
Villamarín, C. P. (2008). Estructura y composición de las comunidades de macroinvertebrados acuáticos en ríos altoandinos del Ecuador y Perú: Diseño de un sistema de medida de la calidad del agua con índices multimétricos (Tesis de doctorado). Universidad de Barcelona, Barcelona.

Vinson, M. (2006). Annotated guide to common aquatic invertebrates. Logan, UT: Utah State University. 